\title{
ON THE DISPLACEMENT BOUNDARY-VALUE PROBLEM OF LINEAR ELASTODYNAMICS*
}

\author{
By M. HAYES (University of East Anglia, Norwich) \\ AND \\ R. J. KNOPS (The University, Newcastle-upon-Tyne)
}

1. Introduction. We are concerned with the uniqueness of the solution for the displacement boundary value problem of linear elastodynamics for a body occupying a closed regular region of space $B$ with smooth surface $\partial B$. Because of the linearity of the equations it suffices to consider the system ${ }^{1}$

$$
\begin{aligned}
c_{i j k l} \frac{\partial^{2} u_{k}}{\partial x_{l} \partial x_{j}} & =\rho \frac{\partial^{2} u_{i}}{\partial t^{2}} \text { in } B, \\
c_{i j k l} & =c_{k l i j},
\end{aligned}
$$

subject to the boundary and initial conditions

$$
\begin{array}{ll}
\mathbf{u}(\mathbf{x}, t)=0, & \mathbf{x} \in \partial B, \quad 0 \leq t<\infty, \\
\mathbf{u}(\mathbf{x}, 0)=\frac{\partial \mathbf{u}}{\partial t}(\mathbf{x}, 0)=0, & \mathbf{x} \in B+\partial B .
\end{array}
$$

Here, $c_{i j k l}$ are the elastic constants, $\mathbf{x}$ is the position vector, $u_{k}(\mathbf{x}, t)$ are the components of the displacement $\mathbf{u}(\mathbf{x}, t), \rho$ is the (positive) density of the elastic body, and time is denoted by $t$. The displacement $\mathbf{u}$ is always assumed to exist and to be real and twice continuously differentiable (i.e. $\mathbf{u} \in C^{2}$ ).

The particular uniqueness problem considered here is to find necessary and sufficient conditions on the $c_{i j k l}$ so that the only twice continuously differentiable solution of (1)-(4) is identically zero. After earlier work on isotropic bodies by Neumann [1] and Gurtin and Sternberg [2], Gurtin and Toupin [3] recently have shown that if

$$
c_{i j k l} A_{i} A_{k} B_{l} B_{j} \geq 0 \text { for all } \mathbf{A}, \mathbf{B} \neq 0
$$

then Eqs. (1)-(4) have only the identically zero solution. Here we show that this result remains true if

$$
c_{i j k l} A_{i} A_{k} B_{l} B_{i}<0 \text { for all } \mathbf{A}, \mathbf{B} \neq 0 .
$$

The method of proof is based upon the uniqueness of analytic functions under Cauchy data.

2. Ellipticity. First we show that (6) is both necessary and sufficient in order that the system (1) is elliptic. ${ }^{2}$

The condition that (1) be elliptic is that

$$
F\left(\varphi^{2}\right) \equiv\left|c_{i j k l} \lambda_{i} \lambda_{l}-\rho \varphi^{2} \delta_{i k}\right| \neq 0,
$$

*Received May 5, 1967; revised manuscript received June 16, 1967.

'Throughout this paper latin indices range over 1,2,3 and summation over repeated suffixes is implied. All equations are referred to Cartesian coordinates $x_{i}$. Bold-face latin minuscules denote vectors.

${ }^{2}$ For definitions of ellipticity, strong-ellipticity, etc. see, e.g., [4]. 
for all real $\lambda$ and $\varphi$ satisfying

$$
\lambda_{i} \lambda_{i}+\varphi^{2} \neq 0 .
$$

On writing

$$
a_{i k}=c_{i j k l} \lambda_{j} \lambda_{l}
$$

it follows from (2) that

$$
a_{i k}=a_{k i}
$$

and thus the zeros $\varphi^{2}$ of $F\left(\varphi^{2}\right)$ are all real. Hence, if $F\left(\varphi^{2}\right) \neq 0$ for real $\varphi$, then the zeros of $F\left(\varphi^{2}\right)$ must all be negative. Thus, necessary and sufficient conditions that (7) hold are

$$
|a|<0, \quad \operatorname{tr} a^{2}-(\operatorname{tr} a)^{2}>0, \quad \operatorname{tr} a<0 .
$$

However, (11) constitute necessary and sufficient conditions that a be negative definite. (See, for instance, [5].) Hence, we conclude that (6) is necessary and sufficient in order that (1) be an elliptic system.

3. Uniqueness. Any solution of class $C^{2}$ to the second order linear elliptic system (1) which has real analytic coefficients consists of real analytic functions in $B$ for $0<$ $t<\infty[6$, p. 136]. We may thus establish the

Theorem. If $c_{i j k l}$ satisfy (6) and (2) then the system (1) subject to (2) and (4) has at most the identically zero solution.

Proof. If (6) is satisfied then the system (1) is elliptic and its solutions are real analytic functions of $\mathbf{x}$ and $t$. Since zero Cauchy data is prescribed on the (noncharacteristic) hypersurface $t=0$, it follows that $\mathbf{u}$ vanishes identically in $B$ for $0 \leq t<\infty$ [7, p. 48].

4. Discussion. When (6) is satisfied the displacement boundary-value problem is, in general, over-determined. For, since solutions are analytic, Cauchy data on the hypersurface $t=0$ determines the solution everywhere so that the boundary data (3) is redundant. Thus, for arbitrarily prescribed boundary and initial data it would appear that even through there is uniqueness of solution, a solution will rarely exist. This may be contrasted with the work of Ericksen [8], [9], [10] who has shown that in general existence fails when uniqueness fails.

Finally, we remark that condition (6) is necessary and sufficient for all plane waves to travel with purely imaginary speeds. Condition (5), which states that the equilibrium equations are semi-strongly-elliptic, implies that all plane waves travel with real or zero speeds. Thus, the displacement boundary value problem is unique provided that either all plane waves travel with purely imaginary speeds or all plane waves travel with real speeds. We do not know whether there is uniqueness when some speeds are real and some are imaginary, i.e. when $c_{i j k l} A_{i} A_{k}$ is indefinite. However, we present here an example to show that in this event it is possible to have a unique solution. We take

so that

$$
c_{i j k l}=\left(-\delta_{i 1} \delta_{k 1}+\delta_{i 2} \delta_{k 2}+\delta_{i 3} \delta_{k 3}\right) \delta_{i l}
$$

Hence,

$$
c_{i j k l}=c_{k l i j} .
$$

$$
c_{i j k l} A_{i} A_{k} B_{l} B_{i}=\left(-A_{1}^{2}+A_{2}^{2}+A_{3}^{2}\right)\left(B_{1}^{2}+B_{2}^{2}+B_{3}^{2}\right) .
$$


Moreover, by (12), equations (1) become:

$$
\begin{gathered}
\partial^{2} u_{1} / \partial x_{i} \partial x_{i}+\rho \partial^{2} u_{1} / \partial t^{2}=0, \\
\partial^{2} u_{\alpha} / \partial x_{i} \partial x_{i}=\rho \partial^{2} u_{\alpha} / \partial t^{2}, \quad(\alpha=2,3) .
\end{gathered}
$$

All solutions of (14) are analytic. In a manner similar to that above, the prescribed zero Cauchy data (4) on the (noncharacteristic) hypersurface $t=0$ shows that $u_{1}$ must be identically zero. The data (3) is redundant. From the uniqueness of the solution to the wave-equation under the data (3) and (4) it follows also that $u_{2}$ and $u_{3}$ are identically zero. Hence, the solution to the present problem is unique.

\section{REFERENCES}

1. F. Neumann, Vorlesungen über die Theorie der Elasticität der festen Körper und des Lichtäthers B. G. Teubner, Leipzig, 1885

2. M. E. Gurtin and E. Sternberg, A note on uniqueness in classical elastodynamics, Quart. Appl. Math. 19, 169 (1961)

3. M. E. Gurtin and R. A. Toupin, A uniqueness theorem for the displacement boundary-value problem of linear elastodynamics, Quart. Appl. Math. 23, 79 (1965)

4. C. B. Morrey, Jr., Contributions to the theory of partial differential equations, Ann. of Math. Studies, Vol. 33, Princeton, 1954, p. 101

5. S. Perlis, Theory of matrices, Addison-Wesley, New York, 1952

6. F. John, Plane waves and spherical means applied to partial differential equations, Interscience, New York, 1955

7. F. John, Partial differential equations (mimeographed lecture notes), N. Y. U., 1952

8. J. L. Ericksen, Non-existence theorems in linear elasticity theory, Arch. Rat. Mech. Anal. 14, 180 (1961)

9. J. L. Ericksen, Non-uniqueness and non-existence in linearised elasticity theory, Contrib. Diff. Eqns. 3, 295 (1964)

10. J. L. Ericksen, Non-existence theorems in linearised elastostatics, J. Diff. Eqns. 1, 446 (1965) 